\title{
Comparison of Surgical Outcomes Between Ab Interno Suture Trabeculotomy and Ab Externo Metal Trabeculotomy in Adult Patients with
}

\section{Glaucoma}

\section{Yasumasa Otori \\ Takanori Matsuoka \\ Miho Kumoi \\ Eri Tachibana \\ Chieko Tsujino \\ Satoshi Matsuda}

Department of Ophthalmology, National Hospital Organization, Osaka National Hospital, Osaka, Japan
Correspondence: Yasumasa Otori Email otori.yasumasa.fe@mail.hosp.go.jp
Purpose: To compare the outcomes of ab interno suture trabeculotomy (AbI-TLO) and ab externo metal trabeculotomy (AbE-TLO) in adult patients with glaucoma aged over 40 years. Patients and Methods: A retrospective chart review was conducted, including adult patients with glaucoma who underwent AbI-TLO or AbE-TLO between January 2015 and June 2019. A single surgeon (YO) performed all the operations. Eighty-one patients (81 eyes) were included in this study. Surgical success was defined as a postoperative intraocular pressure (IOP) of $\leq 18$ $\mathrm{mmHg}$ and an IOP reduction of $\geq 20 \%$ from the preoperative IOP, without requiring additional glaucoma surgery. Success rates were assessed using Kaplan-Meier survival curves and log-rank (Mantel-Cox) tests, while risk factors were analyzed using the Cox proportional hazards model. Results: Forty-nine patients who underwent AbI-TLO and 32 patients who underwent AbETLO were studied; the preoperative IOPs were $27.9 \pm 7.3$ (mean \pm standard deviation) $\mathrm{mmHg}$ and $25.6 \pm 8.1 \mathrm{mmHg}$ in the AbI-TLO and AbE-TLO groups, respectively ( $\mathrm{p}=0.217$ ). The 12-month postoperative IOPs were $15.8 \pm 4.0 \mathrm{mmHg}$ and $16.3 \pm 4.2 \mathrm{mmHg}$ in the AbITLO and AbE-TLO groups, respectively $(\mathrm{p}=0.724)$. The surgical success rates at 12 months were $77.6 \%$ and $62.5 \%$ in the AbI-TLO and AbE-TLO groups, respectively ( $p=0.144$ ). Postoperative hyphema with level formation and ocular hypertension over $30 \mathrm{mmHg}$ were observed in $22.4 \%$ and $26.5 \%$ of patients in the AbI-TLO group and $18.8 \%$ and $12.5 \%$ of those in the AbE-TLO group, respectively. Stepwise multivariate Cox regression analysis showed that a longer axial length was a risk factor for surgical failure (hazard ratio: 2.030; $\mathrm{p}=0.042$ ).

Conclusion: AbI-TLO and AbE-TLO had similar surgical outcomes and postoperative complications. A longer axial length was associated with an insufficient IOP reduction.

Keywords: axial length, gonio-assisted transluminal trabeculotomy, intraocular pressure, MIGS, outcomes, trabeculotomy

\section{Introduction}

Glaucoma is an optic neuropathy characterized by retinal ganglion cell death, leading to both optic nerve head damage and visual field loss. Currently, intraocular pressure (IOP) is the most important determinant for glaucoma treatment, to arrest the progression of visual field loss. ${ }^{1}$

$\mathrm{Ab}$ externo trabeculotomies have been performed since the $1960 \mathrm{~s},{ }^{2,3}$ with the indications for this procedure believed to be congenital, infantile, and juvenile 
glaucoma. ${ }^{4,5}$ In 1977, Luntz et al reported that regardless of the reason, trabeculotomy was not the operation of choice for primary adult-onset open-angle glaucoma (OAG). ${ }^{6}$ Nagata et al expanded the indications for ab externo metal trabeculotomy (AbE-TLO) in Japan to include various types of glaucoma, including child, ${ }^{7,8}$ exfoliation, ${ }^{9}$ primary openangle, ${ }^{9}$ steroid-induced, ${ }^{10}$ and primary angle-closure glaucoma (ACG). ${ }^{11,12}$

In the last ten years, minimally invasive glaucoma surgery (MIGS) for the Schlemm's canal (canal-based MIGS), including ab interno trabeculotomy such as Trabectome, ${ }^{13}$ gonioassisted transluminal trabeculotomy, ${ }^{14}$ the Kahook dual blade, ${ }^{15}$ and Tanito's microhook ${ }^{16}$ procedures were developed with the aim of attenuating physiological aqueous outflow resistance. Trabectome was the first canal-based MIGS that removes a segment of the trabecular meshwork and the inner wall of Schlemm's canal using microelectrocautery. The Kahook dual blade, a slicer-like two blade structure, can also be used for resection in a manner similar to that in Trabectome. On the other hand, gonio-assisted transluminal trabeculotomy and Tanito's microhook can cut the trabecular meshwork and the inner wall of Schlemm's canal in a relatively wider range.

The AbE-TLO procedure requires a conjunctival incision and scleral flap. Japanese glaucoma surgeons prefer making a scleral flap in the inferotemporal region to preserve the upper region for future filtering surgery, if required. According to a survey conducted involving the Japan Glaucoma Society specialists, in 2009, AbE-TLO combined with phacoemulsification was the most common procedure performed for mild open-angle glaucoma with cataract in 2009 (72.7\%). However, the number of canalbased MIGS combined with phacoemulsification surgeries significantly increased in 2019 (79.0\%), compared with number of AbE-TLO surgeries performed (4.6\%). ${ }^{17}$

In this study, we therefore compared AbE-TLO and ab interno suture trabeculotomy (AbI-TLO) performed for the treatment of adult-onset glaucoma in patients aged over 40 years.

\section{Patients and Methods}

This retrospective, single-center, cohort study was approved by the Institutional Review Board of the National Hospital Organization Osaka National Hospital, Osaka, Japan (IRB No. 20121), and followed the tenets of the Declaration of Helsinki. Informed consent was obtained from all patients for inclusion in this study.

\section{Subjects}

Consecutive patients who underwent either AbE-TLO (between January 2015 and February 2017), or AbI-TLO (between February 2017 and June 2019) at the National Hospital Organization Osaka National Hospital were included. All included patients were followed up for at least 12 months. In the present study, the indication for TLO was medically uncontrolled glaucoma, including primary OAG, exfoliation glaucoma, and primary ACG. Patients aged $<40$ years were excluded.

\section{Surgical Techniques}

One surgeon (YO) performed all the surgeries. The surgical procedure for AbE-TLO was as follows: a $4 \times 4 \mathrm{~mm}$ external scleral flap of approximately two-thirds of the scleral thickness was made on the inferotemporal sclera; a $3.5 \times 3.5 \mathrm{~mm}$ internal scleral flap was then made to expose Schlemm's canal. Two metal trabeculotomy probes (Nagata; Inami \& Co., Ltd., Tokyo, Japan) were inserted into Schlemm's canal and rotated by approximately 120 degrees, cutting the inner wall of the trabecular meshwork. Deep sclerectomy and sinusotomy were not performed simultaneously. The external scleral flap and conjunctiva were sutured using 10-0 nylon (MANI Inc., Tochigi, Japan) and 8-0 Vicryl (Ethicon Inc.) sutures, respectively.

AbI-TLO was performed according to Grover's report, ${ }^{14}$ a temporal corneal and upper or lower side ports were created, and a high-molecular-weight viscoelastic material was injected into the anterior chamber through the temporal side port. The patient's head and surgical microscope were oriented to allow suitable visualization of the nasal trabecular meshwork using a Hill open access surgical gonio lens (Ocular Instruments, Bellevue, Washington, United States). The inner wall of the trabecular meshwork was incised using a $27 \mathrm{G}$ needle, and a viscoelastic material was inserted into Schlemm's canal to prevent blood reflux. Rounded tip 5-0 nylon sutures (Chin trabeculotomy sutures; Handaya Co., Ltd. Tokyo, Japan) were inserted into Schlemm's canal from the upper or lower port using 25G forceps (Maxgrip; Alcon Grieshaber, Schaffhausen, Switzerland). After visualizing the nylon suture in Schlemm's canal using a Mori upright surgical gonio lens (Ocular Instruments), the inner wall of Schlemm's canal was gently incised using 25G Maxgrip forceps. The viscoelastic material was washed out with $10 \mathrm{~mL}$ of OPEGURD-MA intraocular irrigating solution (Senju Pharmaceutical Co., Ltd., Osaka, Japan) or BSS 
PLUS solution (Alcon Laboratories, Inc., Geneva, Switzerland) through the temporal side port.

If cataract surgery was simultaneously required, continuous circular capsulorhexis was performed first, followed by AbE-TLO or AbI-TLO, and cataract surgery. The time taken for the surgery was defined as the duration from the first cut to the removal of the surgical drape. All the patients were administered retrobulbar or peribulbar anesthesia. Subsequently, the patients received both $1.5 \%$ topical levofloxacin (NIPRO, Osaka, Japan) and 0.1\% preservative-free betamethasone sodium phosphate eye drops (Nitten, Nagoya, Japan); postoperative eye drops were discontinued 2-4 weeks postoperatively. All the glaucoma drugs were discontinued after surgery and were resumed if the IOP increased. We followed up each patient from one week until one month after surgery, and subsequently at three months postoperatively.

\section{Other Data Collection}

Best-corrected decimal visual acuity (VA) was measured using a Landolt ring chart and was converted to the logarithm of minimal angle resolution (logMAR) for statistical analysis. Data for the IOP was collected at one and seven days; one, two, three, six, nine, and twelve months after the surgery; and at the last visit. The IOP reduction rate was calculated as follows: (preoperative IOP - postoperative IOP at 12 months/preoperative IOP) x 100. The axial length was measured using the IOLMaster 500 (Carl Zeiss Meditec, Germany). The central corneal thickness (CCT) was measured using a specular microscope, TOMEY EM3000 (TOMEY corporation, Nagoya, Japan).

\section{Definitions of Success}

Surgical success was defined as a postoperative IOP of $\leq 18 \mathrm{mmHg}$ and $\geq 20 \%$ reduction from the preoperative IOP, without the requirement for additional glaucoma surgery; the IOP was measured using a Goldmann applanation tonometer (Haag-Streit, Koeniz-Berne, Switzerland).

The glaucoma drug scores were as follows: (1) the use of glaucoma eye drops contributed one point, while (2) a fixed combination and the use of an oral carbonic anhydrase inhibitor contributed two points. ${ }^{18}$

\section{Statistical Analysis}

Success rates were assessed using Kaplan-Meier survival curves and Log-rank (Mantel-Cox) tests; if the IOP went beyond the success range twice in a row, the first event was defined a surgical failure. Other quantifiable parameters were compared using the Mann-Whitney $U$-test. The chi-square test was used for noncontinuous variables with $\mathrm{n} \geq 5$; Fisher's exact test was used for noncontinuous variables with $\mathrm{n}<5$. Kaplan-Meier survival curves and Log rank tests were performed using Cox proportional hazard analyses. Factors with P-values $<0.1$ in the univariate Cox regression analysis underwent stepwise multivariate Cox regression analysis. All statistical analyses were performed using SYSTAT (version 13.1; HULINKS, Tokyo, Japan). The level of statistical significance was set at $\mathrm{p}<0.05$.

\section{Results}

The demographic characteristics of the patients are presented in Table 1. Forty-nine and 32 patients who underwent AbITLO and AbE-TLO, respectively, were studied. The

Table I Patient Demographic Data

\begin{tabular}{|c|c|c|c|}
\hline & AbE-TLO (n=32) & Abl-TLO (n=49) & P-value \\
\hline Age (years) & $66.5 \pm 10.6(46-80)$ & $72.9 \pm 9.6(51-91)$ & 0.006 \\
\hline Female/Male & $19 / 13$ & $26 / 23$ & 0.762 \\
\hline ACG/OAG (rates of ACG) & $21 / 11(65.6 \%)$ & $17 / 32$ (34.7\%) & 0.012 \\
\hline Preoperative IOP (mmHg) & $25.6 \pm 8.1(17-43)$ & $27.9 \pm 7.3(15-52)$ & 0.217 \\
\hline Preoperative glaucoma drug score & $3.7 \pm 1.2(2-6)$ & $4.2 \pm 1.5(2-7)$ & 0.094 \\
\hline Preoperative LogMAR visual acuity & $0.08 \pm 0.26(0.00-1.40)$ & $0.22 \pm 0.32(0.00-1.52)$ & 0.003 \\
\hline Rates of combined cataract surgery & $87.5 \%(28 / 32)$ & $63.2 \%(31 / 49)$ & 0.012 \\
\hline Rates of pseudophakic eyes & $0 \%$ & $34.7 \%(17 / 49)$ & $<0.0001$ \\
\hline CCT $(\mu \mathrm{m})$ & $526 \pm 28(467-565)$ & $513 \pm 31(464-568)$ & 0.064 \\
\hline Axial length $(\mathrm{mm})$ & $23.39 \pm 1.59(21.49-29.51)$ & $23.69 \pm 2.01(20.59-31.73)$ & 0.526 \\
\hline Follow-up periods (months) & $33.2 \pm 19.4(12.5-69.0)$ & $22.0 \pm 9.6(\mid 2.2-40.2)$ & 0.002 \\
\hline
\end{tabular}

Abbreviations: AbE-TLO, ab externo metal trabeculotomy; AbI-TLO, ab interno suture trabeculotomy; IOP, intraocular pressure; LogMAR, the logarithm of minimal angle resolution; CCT, central corneal thickness. 
preoperative IOP and glaucoma drug scores, sex, CCT, and axial length were not significantly different between the AbITLO group and the AbE-TLO group; however, the age of the patients in the AbI-TLO group was significantly higher than that of those in the AbE-TLO group. Additionally, the rates of ACG combined with cataract extraction procedure were significantly higher in the AbE-TLO group than in the AbITLO group. The prevalence of pseudophakic eyes was significantly higher in the AbI-TLO group than in the AbE-TLO group. The preoperative logMAR VA of the AbE-TLO group was significantly better than that of the AbI-TLO group. The total follow-up periods in the AbE-TLO group were significantly longer than those of the AbI-TLO group. It was determined that $93.8 \%$ (76/81) of the patients had pseudophakic eyes. No patients had previously undergone selective laser trabeculoplasty (SLT) in the present study.

Postoperative outcomes and adverse effects are presented in Table 2. The duration of surgery of the AbI-TLO group $(34.9 \pm 7.8, \mathrm{n}=31)$ was significantly shorter than that of the AbE-TLO group $(50.4 \pm 8.0, \mathrm{n}=28 ; \mathrm{p}<0.0001)$. The cut ratio for Schlemm's canal was $212.2 \pm 65.6$ (120-360) degrees in the AbI-TLO group and $116.3 \pm 14.8(60-120)$ degrees in the $\mathrm{AbE}$ group; the preoperative IOP was $27.9 \pm 7.3 \mathrm{mmHg}$ in the AbI-TLO group and $25.6 \pm 8.1 \mathrm{mmHg}$ in the AbETLO group $(\mathrm{p}=0.217)$. The postoperative IOP at 12 months of follow-up was $15.8 \pm 4.0 \mathrm{mmHg}$ in the AbI-TLO group and $16.3 \pm 4.2 \mathrm{mmHg}$ in the AbE-TLO group ( $\mathrm{p}=0.724)$. The IOP reduction rate at 12 months was $39.7 \pm 20.3 \%$ in the AbI-TLO group and $33.6 \pm 17.8 \%$ in the AbE-TLO group $(p=0.067)$. Further, the postoperative glaucoma drug score at the final visit was $3.4 \pm 1.5$ in the AbI-TLO group and 2.9 \pm 1.4 in the AbE-TLO group $(\mathrm{p}=0.223)$. The postoperative glaucoma drug score was significantly decreased in both the
AbI-TLO group ( $\mathrm{p}=0.007$, paired $t$-test) and the AbE-TLO group ( $\mathrm{p}=0.004$, paired $t$-test) compared with the respective preoperative glaucoma drug scores. The postoperative logMAR VA in the AbI-TLO group was significantly better than the preoperative $\log$ MAR VA $(\mathrm{p}=0.007$, paired $t$-test); however, no significant changes were observed in the postoperative $\log$ MAR VA of AbE-TLO as compared to preoperative $\log$ MAR VA $(\mathrm{p}=0.175$, paired $t$-test $)$.

The patients' postoperative IOPs reduced significantly after TLO (Figures 1 and 2). The surgical success rate according to Kaplan-Meier analysis was $47.1 \%$ at 34 months after TLO (Figure 3), whereas the surgical success rates at 12 months postoperatively were $77.6 \%$ and $62.5 \%$ in the AbI-TLO and AbE-TLO groups, respectively $(\mathrm{p}=0.144)$. According to the Kaplan-Meier survival curves, the success rates of AbI-TLO and AbE-TLO were not significant (Figure 4, Log-rank test; $\mathrm{p}=0.875$ ); moreover, the success rates of ACG $(n=38)$ and OAG $(n=43)$ were not significant (Log-rank test; $\mathrm{p}=0.299$ ). Postoperative hyphema with level formation and transient ocular hypertension over $30 \mathrm{mmHg}$ were observed in $22.4 \%$ and $26.5 \%$ of patients in the AbI-TLO group, and $18.8 \%$ and $12.5 \%$ of those in the AbE-TLO group, respectively.

Stepwise multivariate Cox regression analysis showed that a longer axial length was a risk factor for surgical failure (hazard ratio: 2.030; $\mathrm{p}=0.042$, Table 3 ); eyes with an axial length $<24.0 \mathrm{~mm}$ thus exhibited significantly better outcomes than those with an axial length $\geq 24.0 \mathrm{~mm}$ (Figure 5, Log-rank test; $\mathrm{p}=0.031$ ).

\section{Discussion}

Our study revealed that the success rates and postoperative complications between AbI-TLO and AbE-TLO were not

Table 2 Postoperative Outcomes and Adverse Effects

\begin{tabular}{|l|l|l|l|}
\hline & AbE-TLO (n=32) & AbI-TLO (n=49) & P-value \\
\hline Preoperative IOP (mmHg) & $25.6 \pm 8.1(I 7-43)$ & $27.9 \pm 7.3(I 5-52)$ & 0.217 \\
Postoperative IOP at I2 months (mmHg) & $16.3 \pm 4.2(I I-30)$ & $15.8 \pm 4.0(5-26)$ & 0.724 \\
IOP reduction rate at I2 months (\%) & $33.6 \pm 17.6(-13.6-72 . I)$ & $39.7 \pm 20.3(-4.5-67.3)$ & 0.067 \\
Postoperative IOP at last visit (mmHg) & $17.8 \pm 6.4(I I-33)$ & $15.9 \pm 4.2(5-30)$ & 0.338 \\
Preoperative glaucoma drug score & $3.7 \pm 1.2(2-6)$ & $4.2 \pm 1.5(2-7)$ & 0.094 \\
Postoperative glaucoma drug score (last visit) & $2.9 \pm 1.4(0-6)$ & $3.4 \pm 1.5(0-6)$ & 0.223 \\
Postoperative LogMAR visual acuity & $0.06 \pm 0.27(0.00-1.52)$ & $0.15 \pm 0.30(0.00-1.52)$ & 0.057 \\
Operation time (min) & $48.8 \pm 8.8(33.0-82.0)$ & $30.4 \pm 9.2(15.0-60.0)$ & $<0.0001$ \\
Hyphema with level formation & $18.8 \%(6 / 32)$ & $22.4 \%(I I / 49)$ & 0.607 \\
Transient IOP spike over 30 mmHg & $12.5 \%(4 / 32)$ & $26.5 \%(I 3 / 49)$ & 0.164 \\
Success rates at I2 months & $62.5 \%(20 / 32)$ & $77.6 \%(38 / 49)$ & 0.328 \\
\hline
\end{tabular}

Abbreviations: AbE-TLO, ab externo metal trabeculotomy; Abl-TLO, ab interno suture trabeculotomy; IOP, intraocular pressure. 


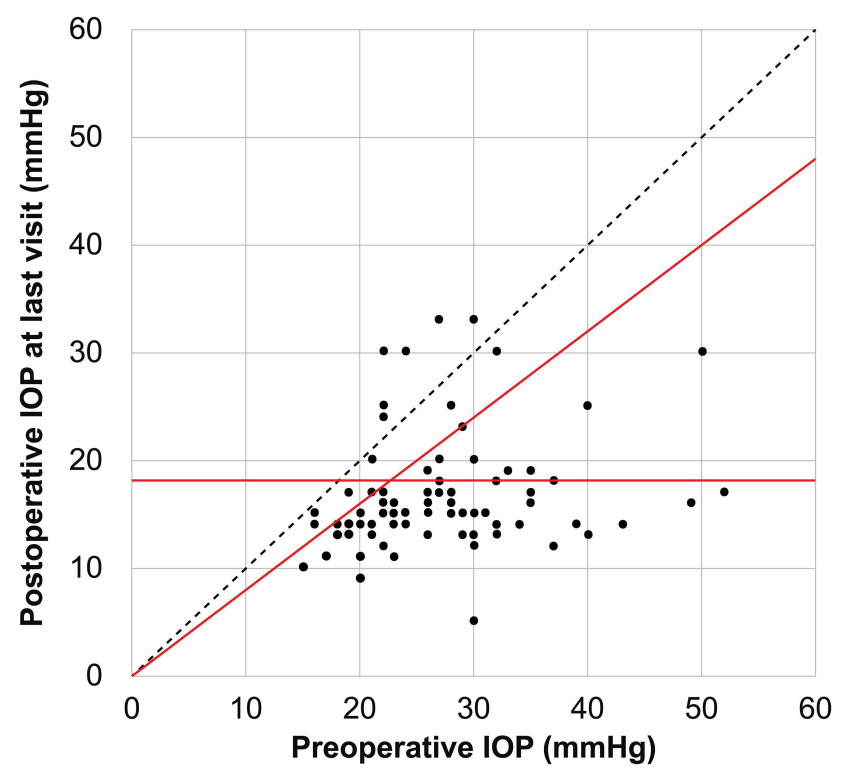

Figure I Scattergram of preoperative and postoperative intraocular pressure (IOP) at the final visit in all eyes. Each point represents one eye with the preoperative IOP as the abscissa and the postoperative IOP as the ordinate. The horizontal red line indicates $18 \mathrm{mmHg}$, and the oblique red line indicates a $20 \%$ reduction from the preoperative IOP.

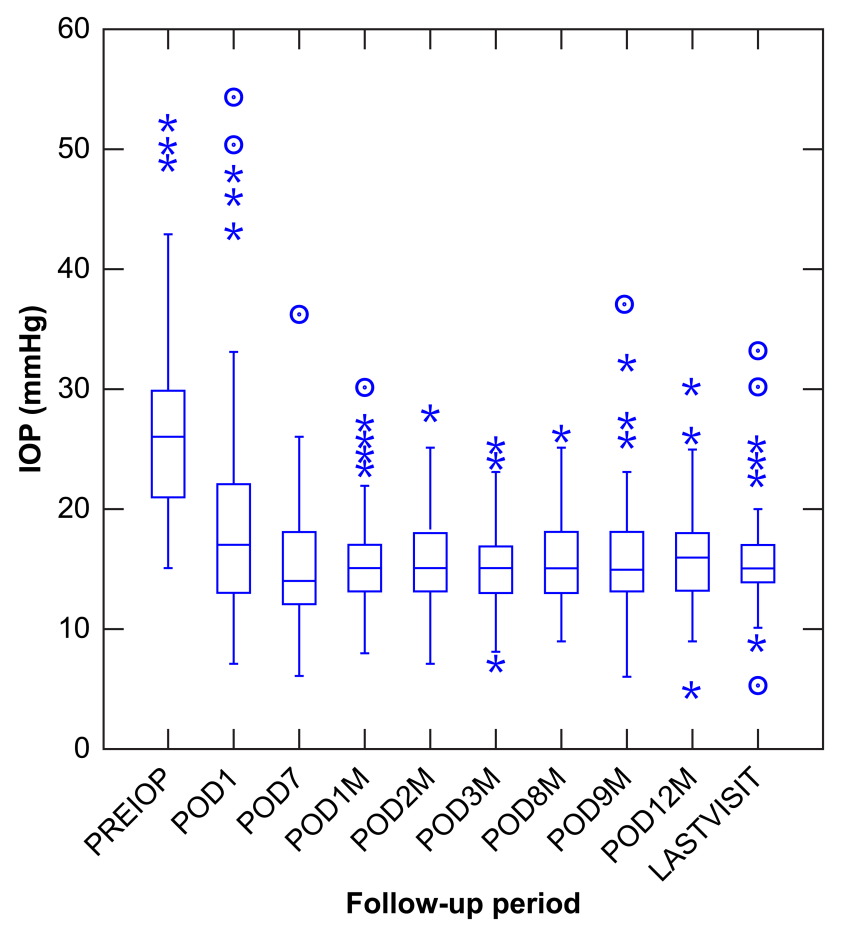

Figure 2 Box plot of the intraocular pressure (IOP) and observation time in all eyes. The box plots show the median, and 25 th and 75 th percentiles of the data. Asterisks and circles denote outliers. The graph shows a significant reduction in IOP after trabeculotomy (TLO). Number of eyes were as follows: PREOPE $(n=8 I)$, PODI $(n=8 I)$, POD7 $(n=8 I)$, PODIM $(n=8 I)$, POD2M $(n=76)$, POD3M $(n=77)$, POD6M $(n=79)$, POD9M $(n=79)$, PODI2M $(n=79)$, and LASTVISIT $(n=8 I)$.

Abbreviations: POD, postoperative day; $M$, month(s).

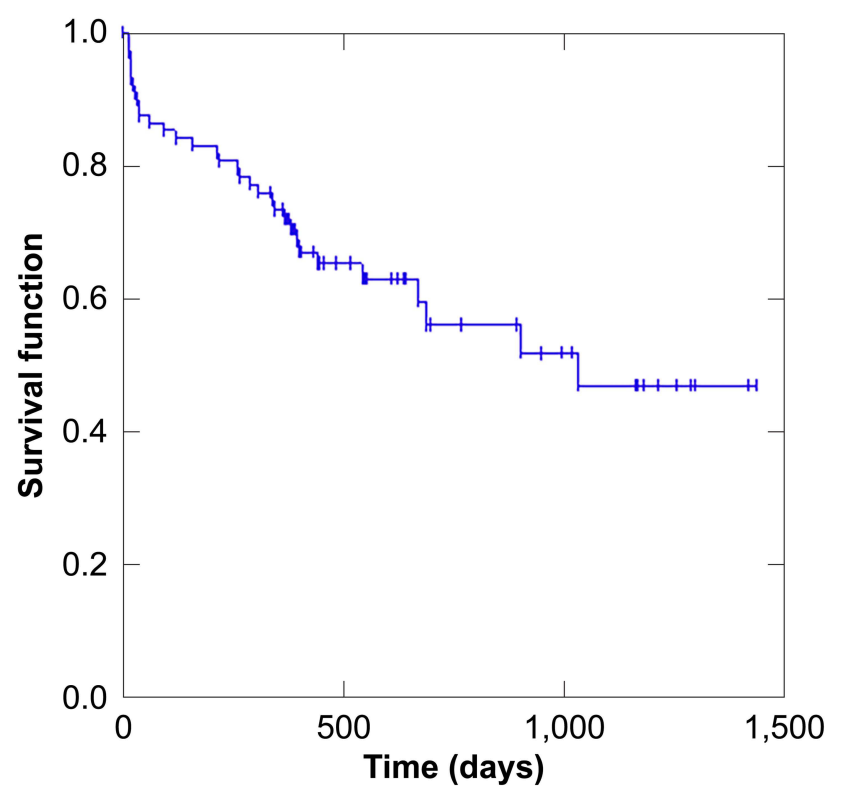

Figure 3 Results of Kaplan-Meier survival curve analysis in all eyes. The definition of surgical success was a postoperative intraocular pressure (IOP) of $\leq 18 \mathrm{mmHg}$ and $\geq 20 \%$ reduction in from the preoperative IOP, without additional glaucoma surgery.

significantly different. We also found that a longer axial length was associated with insufficient IOP reduction for TLO.

Kinoshita-Nakano et al reported that at 12 months postoperatively, the IOP reduction rate was higher for AbE-TLO combined with deep sclerectomy/sinusotomy (30.9\%. Preoperative IOP $24.2 \pm 6.6 \mathrm{mmHg}$, preoperative glaucoma drug score $3.2 \pm 0.8$ ) than for AbI-TLO using a Trabectome (23.0\%. Preoperative IOP $22.6 \pm 7.4 \mathrm{mmHg}$, preoperative glaucoma drug score $3.5 \pm 1.0) .{ }^{19}$ They also showed that neither combined cataract surgery nor the type of surgery were significantly related to surgical success based on the definition of success used in this study, suggesting that successful surgical outcomes following Trabectome use and AbE-TLO combined with deep sclerectomy/sinusotomy were not significantly different after 36 months of follow-up. Mori et al reported that the success rates of AbE-TLO combined with deep sclerectomy and AbI-TLO using a microhook were not significantly different at the 12-month follow-up. ${ }^{18}$ In their study, the definition of surgical success was a postoperative IOP $\leq 21 \mathrm{mmHg}$ and $\geq 20 \%$ reduction in IOP. They reported that the IOP reduction rate for AbI-TLO using a microhook $(28.4 \pm 7.8 \mathrm{mmHg}, 4.9 \pm 1.1)$ and AbE-TLO with deep sclerectomy (preoperative IOP $32.5 \pm 11.2 \mathrm{mmHg}$, preoperative glaucoma drug score $4.3 \pm 1.4$ ) was $34.4 \%$ and $40.5 \%$ at 12 months after surgery, respectively. In this 


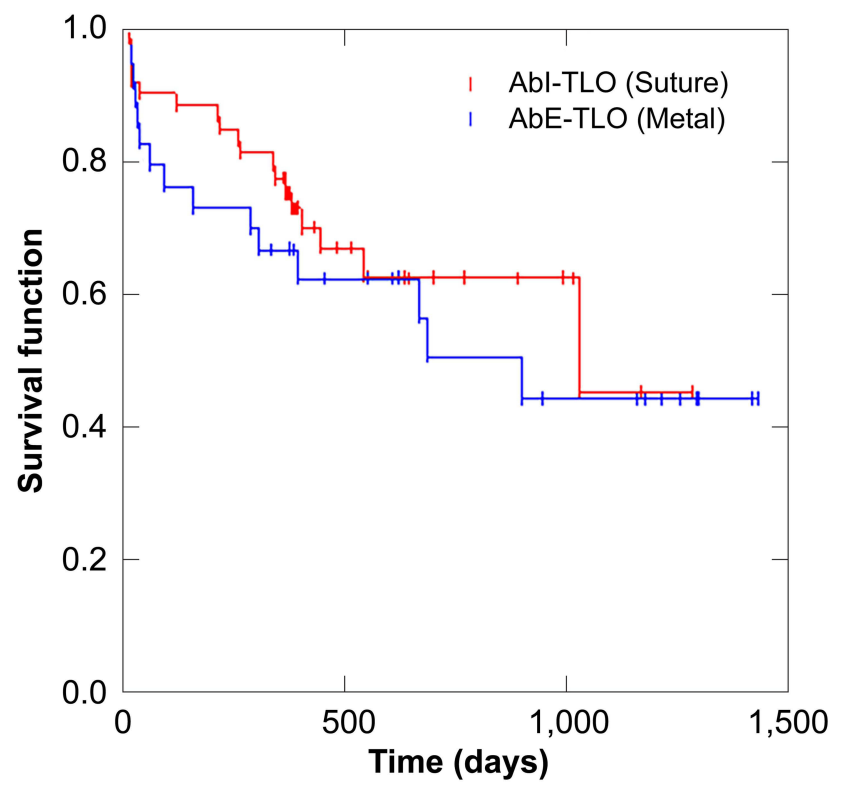

Figure 4 Results of Kaplan-Meier survival curve analysis for both procedures. There were no significant differences in success rates between ab interno trabeculotomy (Abl-TLO, red line) and ab externo trabeculotomy (AbE-TLO, blue line, Log-rank test; $\mathrm{p}=0.875$ ).

study, the IOP reduction rate for AbI-TLO $(27.9 \pm 7.3$ $\mathrm{mmHg}, 4.2 \pm 1.5$ ) and AbE-TLO (preoperative IOP 25.6 $\pm 8.1 \mathrm{mmHg}$, preoperative glaucoma drug score $3.7 \pm 1.2$ ) was $39.7 \%$ and $33.6 \%$ at 12 months after surgery, respectively. The IOP reduction rate at 12 months of AbI-TLO using 5-0 nylon suture seems to be better than that of AbITLO using a Trabectome or a microhook. In summary, the efficacy of AbI-TLO and AbE-TLO were equivalent in terms of IOP reduction.

Ahuja et al reported that when the definition of surgical success was a postoperative IOP $\leq 21 \mathrm{mmHg}$, or $\geq 20 \%$ reduction of IOP, cases of AbI-TLO by Trabectome use combined with cataract surgery had a higher rate of

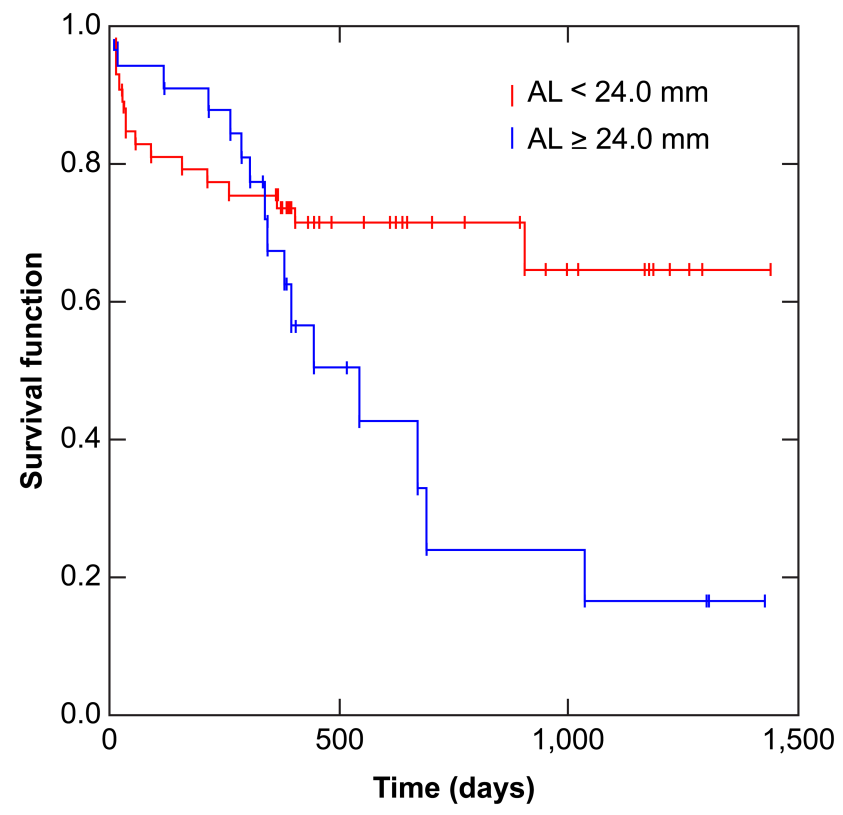

Figure 5 Results of Kaplan-Meier survival curve analysis stratified according to the axial length of $24.0 \mathrm{~mm}$. The axial length was $<24.0 \mathrm{~mm}$ (red line) or $\geq 24.0 \mathrm{~mm}$ (blue line). Eyes with an axial length $<24.0 \mathrm{~mm}$ had significantly better outcomes than eyes with an axial length $\geq 24.0 \mathrm{~mm}$ (Log-rank test; $\mathrm{p}=0.03 \mathrm{I}$ ).

Abbreviation: $A L$, axial length.

success after surgery than cases of AbI-TLO by Trabectome use only. ${ }^{20}$ However, if the definition of surgical success was a postoperative IOP $\leq 18 \mathrm{mmHg}$ and $\geq 20 \%$ reduction (the same criteria as in this study), the success rates decreased, along with the differences in the success rates between AbI-TLO combined with cataract surgery and AbI-TLO alone. In this study, the rate of combined cataract surgery was $72 \%$ and thus unfavorably influenced surgical success; conversely, the efficacy of ACG and OAG for TLO was equivalent. We compared the success rate of TLO combined with cataract surgery $(n=59)$ and TLO alone for pseudophakia $(n=17)$ and

Table 3 Results of Univariate and Multivariate Analyses

\begin{tabular}{|c|c|c|c|c|c|c|}
\hline \multirow[t]{2}{*}{ Parameter } & \multicolumn{3}{|l|}{ Univariate } & \multicolumn{3}{|l|}{ Multivariate } \\
\hline & Hazard Ratio & $95 \% \mathrm{Cl}$ & $P$ value & Hazard Ratio & $95 \% \mathrm{Cl}$ & $P$ value \\
\hline Age & 1.194 & $1.154-1.234$ & 0.232 & - & - & - \\
\hline Sex (Female) & 1.576 & $0.796-2.356$ & 0.115 & - & - & - \\
\hline CCT & 1.956 & $1.942-1.970$ & 0.050 & - & - & - \\
\hline Preoperative medications & 1.288 & $0.940-1.636$ & 0.198 & - & - & - \\
\hline AbE-TLO & 0.619 & $-0.235-1.473$ & 0.536 & - & - & - \\
\hline Combined cataract surgery & 1.701 & $-0.053-3.455$ & 0.089 & - & - & - \\
\hline OAG & 1.920 & $1.181-2.70 \mid$ & 0.052 & - & - & - \\
\hline $\mathrm{AL}$ & 2.018 & $1.826-2.210$ & 0.044 & 2.030 & $1.886-2.174$ & 0.042 \\
\hline Pseudophakia & 0.319 & $-1.649-1.287$ & 0.749 & - & - & - \\
\hline
\end{tabular}

Abbreviations: CCT, central corneal thickness; AbE-TLO, ab externo metal trabeculotomy; OAG, open-angle glaucoma; AL, axial length; Cl, confidence interval. 
phakia $(n=5)$. The TLO-only group included several patients with pseudophakic eyes. The outcomes of TLO combined with cataract surgery were similar to those of TLO alone. These findings suggest that AbI- or AbE-TLO might have similar IOP-lowering effects for phakic and pseudophakic eyes.

In the present study, the cut ratio of the inner wall of Schlemm's canal in the AbI-TLO group was wider than that in the AbE-TLO group; nevertheless, the success rates were not significantly different between the two groups. Manabe et al reported that during AbE-TLO (suture), incisions in Schlemm's canal of $\geq 150$ degrees did not affect the reduction in IOP at 12 months postoperatively. ${ }^{21}$ Sato et al also reported that incisions in different locations and ranges of Schlemm's canal during AbI-TLO did not affect surgical outcomes at the 12 month follow-up. ${ }^{22}$ These findings suggest that incisions in Schlemm's canal of $>120$ degrees may be sufficient to reduce IOP during short-term follow-up periods.

We also demonstrated that a longer axial length was related to insufficient IOP reduction for TLO. Kuusniemi et al reported that an axial length $>23.82 \mathrm{~mm}$ might reduce Trabectome procedure success, ${ }^{22}$ however, the mechanism of IOP elevation after TLO in patients with a longer axial length remains unclear. Canal-based MIGS is expected to increase the outflow pathway from the trabecular meshwork to the collector channel. If the main outflow resistance were distal to the collector channel, canal-based MIGS may be ineffective; thus, a longer axial length may cause elongation of the aqueous vein, thereby increasing aqueous venous pressure. Kuusniemi et al reported that a history of SLT before canal-based MIGS was a risk factor for surgical failure, ${ }^{23,24}$ and while it is easy to recognize that canal-based MIGS might not be effective in patients resistant to SLT, no studies have reported a longer axial length to be a risk factor for SLT failure; therefore, further investigation is required.

AbI-TLO requires a clear corneal incision, which is beneficial for future filtering surgery; however, AbETLO requires a conjunctival incision and scleral flap. Thus, the duration of AbI-TLO is significantly shorter than that of AbE-TLO. AbI-TLO was performed to directly observe the gonio using a surgical gonio lens. First, we identified the scleral spur, subsequently making an incision on the borderline of the pigmented and nonpigmented trabecular meshwork; if a white band-representative of the underlying sclera-could be seen, it was easy to set a 5-0 nylon suture into Schlemm's canal. Nylon sutures may not hurt the outer wall of Schlemm's canal; therefore, the risk of intraoperative complications during the AbE-TLO procedure - such as early perforation of the trabeculotome probe, cyclodialysis, iridodialysis, and Descemet's membrane rupture - may be lower in the AbI-TLO procedure. Thus, the AbI-TLO procedure reduces the duration of the surgery and is minimally invasive when compared with AbE-TLO.

The limitations of this study include its retrospective nature, the possibility of selection bias, relatively shortterm follow-up, and limited sample size. Canal-based MIGS is believed to be safer than glaucoma filtering surgery; however, the postoperative IOP in canal-based MIGS is higher than that of glaucoma filtering surgery. Ophthalmologists should, therefore, reconsider the indications for canal-based MIGS, even in patients with early stage glaucoma; a larger study may identify more preoperative factors influencing the outcomes of canal-based MIGS.

\section{Conclusions}

In conclusion, AbI-TLO reduces the duration of surgery and is minimally invasive when compared with AbE-TLO, and the success rates and postoperative complications between AbI-TLO and AbE-TLO were equivalent throughout the short-term follow-up periods. Additionally, a longer axial length was associated with insufficient IOP reduction in TLO.

\section{Abbreviations}

AbE-TLO, ab externo metal trabeculotomy; AbI-TLO, ab interno suture trabeculotomy; ACG, angle-closure glaucoma; IOP, intraocular pressure; OAG, open-angle glaucoma; SLT, Selective laser trabeculoplasty; canal-based MIGS, minimally invasive glaucoma surgery for Schlemm's canal.

\section{Acknowledgments}

We would like to thank Editage (www.editage.com) for English language editing.

\section{Funding}

The authors received no funding for the preparation of this manuscript.

\section{Disclosure}

The authors report no conflicts of interest in the conduct of this work. 


\section{References}

1. Weinreb RN, Khaw PT. Primary open-angle glaucoma. Lancet. 2004;363(9422):1711-1720. doi:10.1016/S0140-6736(04)16257-0

2. Burian HM. A case of Marfan's syndrome with bilateral glaucoma. With description of a new type of operation for developmental glaucoma (trabeculotomy ab externo). Am $J$ Ophthalmol. 1960;50:1187-1192. doi:10.1016/0002-9394(60)91007-2

3. Smith R. A New technique for opening the canal of schlemm: preliminary report. Br J Ophthalmol. 1960;44(6):370-373. doi:10.1136/ bjo. 44.6 .370

4. Harms H, Dannheim R. Trabeculotomy-results and problems. Bibl Ophthalmol. 1970;81:121-131.

5. McPherson SD Jr. Results of external trabeculotomy. Am $J$ Ophthalmol. 1973;76(6):918-920. doi:10.1016/0002-9394(73) 90081-0

6. Luntz MH, Livingston DG. Trabeculotomy ab externo and trabeculectomy in congenital and adult-onset glaucoma. Am J Ophthalmol. 1977;83(2):174-179. doi:10.1016/0002-9394(77)90612-2

7. Akimoto M, Tanihara H, Negi A, Nagata N. Surgical results of trabeculotomy ab externo for developmental glaucoma. Arch Ophthalmol. 1994;112(12):1540-1544. doi:10.1001/ archopht.1994.01090240046024

8. Ikeda $\mathrm{H}$, Ishigooka $\mathrm{H}$, Muto $\mathrm{T}$, Tanihara $\mathrm{H}$, Nagata M. Long-term outcome of trabeculotomy for the treatment of developmental glaucoma. Arch Ophthalmol. 2004;122(8):1122-1128. doi:10.1001/ archopht.122.8.1122

9. Tanihara H, Negi A, Akimoto M, et al. Surgical effects of trabeculotomy ab externo on adult eyes with primary open angle glaucoma and pseudoexfoliation syndrome. Arch Ophthalmol. 1993;111 (12):1653-1661. doi:10.1001/archopht.1993.01090120075025

10. Honjo M, Tanihara H, Inatani M, Honda Y. External trabeculotomy for the treatment of steroid-induced glaucoma. J Glaucoma. 2000;9 (6):483-485. doi:10.1097/00061198-200012000-00011

11. Tanihara H, Negi A, Akimoto M, Nagata M. Long-term results of non-filtering surgery for the treatment of primary angle-closure glaucoma. Graefes Arch Clin Exp Ophthalmol. 1995;233 (9):563-567. doi:10.1007/BF00404707

12. Kiuchi Y, Tsujino C, Nakamura T, Otori Y, Mochizuki H. Phacoemulsification and trabeculotomy combined with goniosynechialysis for uncontrollable chronic angle-closure glaucoma. Ophthalmic Surg Lasers Imaging. 2010;41(3):348-354. doi:10.3928/15428877-20100430-09
13. Minckler DS, Baerveldt G, Alfaro MR, Francis BA. Clinical results with the trabectome for treatment of open-angle glaucoma. Ophthalmology. 2005;112(6):962-967. doi:10.1016/j.ophtha.2004.12.043

14. Grover DS, Godfrey DG, Smith O, Feuer WJ, Montes de Oca I, Fellman RL. Gonioscopy-assisted transluminal trabeculotomy, ab interno trabeculotomy: technique report and preliminary results. Ophthalmology. 2014;121(4):855-861. doi:10.1016/j. ophtha.2013.11.001

15. Seibold LK, Soohoo JR, Ammar DA, Kahook MY. Preclinical investigation of ab interno trabeculectomy using a novel dual-blade device. Am J Ophtahlmol. 2013;155(3):524-529. doi:10.1016/j.ajo.2012.09. 023

16. Tanito M. Microhook ab interno trabeculotomy, a novel minimally invasive glaucoma surgery. Clin Ophthalmol. 2017;12:43-48. doi:10.2147/OPTH.S152406

17. Iwasaki K, Arimura S, Takamura Y, Inatani M. Clinical practice preferences for glaucoma surgery in Japan: a survey of Japan Glaucoma Society specialists. Jpn $J$ Ophthalmol. 2020;64 (4):385-391. doi:10.1007/s10384-020-00749-w

18. Mori S, Murai Y, Ueda K, et al. A comparison of the 1-year surgical outcomes of ab externo trabeculotomy and microhook ab interno trabeculotomy using propensity score analysis. BMJ Open Ophthalmol. 2020;5(1):e000446. doi:10.1136/bmjophth-2020-000446

19. Kinoshita-Nakano E, Nakanishi H, Ohashi-Ikeda H, Morooka S, Akagi T. Comparative outcomes of trabeculotomy ab externo versus trabecular ablation ab interno for open angle glaucoma. Jpn J Ophthalmol. 2018;62(2):201-208. doi:10.1007/s10384-017-0559-0

20. Ahuja Y, Ma Khin Pyi S, Malihi M, Hodge DO, Sit AJ. Clinical results of ab interno trabeculotomy using the trabectome for open-angle glaucoma: the Mayo Clinic series in Rochester, Minnesota. Am J Ophthalmol. 2013;156(5):927-935.

21. Manabe SI, Sawaguchi S, Hayashi K. The effect of the extent of the incision in the schlemm canal on the surgical outcomes of suture trabeculotomy for open-angle glaucoma. Jpn J Ophthalmol. 2017;61 (1):99-104. doi:10.1007/s10384-016-0487-4

22. Sato T, Kawaji T. 12-month randomised trial of $360^{\circ}$ and $180^{\circ}$ Schlemm's canal incisions in suture trabeculotomy ab interno for open-angle glaucoma. Br J Ophthalmol. 2021;105(8):1094-1098.

23. Kuusniemi AM, Lindbohm N, Allinen P, Koskinen M, Harju M. Ab interno trabeculotomy: key prognostic factors. J Glaucoma. 2020;29 (3):211-216. doi:10.1097/IJG.0000000000001432

24. Kasahara M, Shoji N. Effectiveness and limitations of minimally invasive glaucoma surgery targeting schlemm's canal. Jpn J Ophthalmol. 2021;65(1):6-22. doi:10.1007/s10384-020-00781-w
Clinical Ophthalmology

\section{Publish your work in this journal}

Clinical Ophthalmology is an international, peer-reviewed journal covering all subspecialties within ophthalmology. Key topics include: Optometry; Visual science; Pharmacology and drug therapy in eye diseases; Basic Sciences; Primary and Secondary eye care; Patient Safety and Quality of Care Improvements. This journal is indexed on PubMed
Central and CAS, and is the official journal of The Society of Clinical Ophthalmology (SCO). The manuscript management system is completely online and includes a very quick and fair peer-review system, which is all easy to use. Visit http://www.dovepress.com/ testimonials.php to read real quotes from published authors.

\section{Dovepress}

